RESEARCH ARTICLE

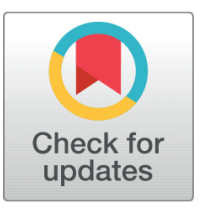

6 OPEN ACCESS

Received: 04-05-2020

Accepted: 12-05-2020

Published: 18-06-2020

Editor: Dr. Natarajan Gajendran

Citation: Rameshbabu R, Suresh GR (2020) Design of adaptive feedback control for new 3D chaotic system and its digital implementation on FPGA. Indian Journal of Science and Technology 13(20): 1977-1990. https ://doi.org/10.17485/IJST/v13i20.498

\section{*Corresponding author.}

R Rameshbabu

Research Scholar, Department of Electronics and Communication Engineering, St. Peter's Institute of Higher Education and Research, Chennai, TN, India.

Tel.: +91-902-504-1638

rrameshbabu15@gmail.com

\section{Funding: None}

Competing Interests: None

Copyright: () 2020 Rameshbabu, Suresh. This is an open access article distributed under the terms of the Creative Commons Attribution License, which permits unrestricted use, distribution, and reproduction in any medium, provided the original author and source are credited.

Published By Indian Society for Education and Environment (iSee)

\section{Design of adaptive feedback control for new 3D chaotic system and its digital implementation on FPGA}

\author{
R Rameshbabu ${ }^{1 *}$, G R Suresh ${ }^{2}$ \\ 1 Research Scholar, Department of Electronics and Communication Engineering, St. Peter's \\ Institute of Higher Education and Research, Chennai, TN, India. Tel.: +91-902-504-1638 \\ 2 Professor, Department of Biomedical Engineering, St. Peter's Institute of Higher Education \\ and Research, Chennai, TN, India
}

\section{Abstract}

Background/Objectives : In this research work, digital circuit implementation on FPGA of an adaptive feedback control methodology for a new 3 - D chaotic system is proposed Methods/Statistical analysis: The chaos synchronization is achieved using adaptive feedback control method. The new adaptive controllers are designed to achieve the chaos synchronization for the identical new chaotic system. The FPGA implementation of chaos synchronization using numerical methods induces artificial suppression in the chaotic system or chaotic behavior can be dead in very short-time. In this research work, the FPGA implementation of chaos synchronization is achieved with the help of automatic code generator like System generator in Matlab simulink. The adaptive feedback control for identical new chaotic system is coded with VHDL with 32 bit fixed point number, 12 for the entire and 20 for the fraction. Findings: In this paper, we designed a new 3D chaotic system and its chaotic behavior is verified using Lyapunov exponents, stability analysis and Poincare map. The complete synchronization for proposed chaotic system is achieved using adaptive feedback control methodology. The digital circuit realization of adaptive feedback control for the synchronization of identical chaotic system based on FPGA is achieved for the various applications of digital information systems. Simulation results and FPGA outputs illustrate the effectiveness of our proposed method. Novelty/Applications: The digital implementation of adaptive feedback control has many engineering applications such as digital data transmission, digital modulation, video encryption, digital cryptosystem etc.

Keywords: Chaotic system; complete synchronization; adaptive feedback control; FPGA implementation; digital implementation 


\section{Introduction}

Chaotic systems are nonlinear dynamical systems which are highly sensitive to initial conditions. This sensitivity is popularly known as the butterfly effect. Chaos is an interesting nonlinear phenomenon and has been studied well in the last three decades. Chaos theory has wide applications in several fields like oscillators ${ }^{(1,2)}$, image encryption ${ }^{(3,4)}$, chemical reactors ${ }^{(5,6)}$, secure communications ${ }^{(7,8)}$, biological systems ${ }^{(9,10)}$, etc. In this paper a new $3 \mathrm{D}$ chaotic system is introduced and its basic properties such as Lyapunnov exponents, stability analysis, Poincare map are studied. The new chaotic system introduced in this paper consists of four nonlinear terms and five parameters unstable at all equilibrium points.

Still the chaos synchronization is a nontrivial task interesting impact on chaos based engineering applications such as secure communications etc. In last two decades many researchers presented the chaos synchronization using master - slave methodology ${ }^{(11-29)}$. In this methodology, particular chaotic system is considered as master system and another or same chaotic system can be considered as the slave system. The chaos synchronization can be achieved if the output of the slave system tracks the output of the master system asymptotically. In the last two decades, various schemes have been successfully applied for chaos synchronization such as OGY method, active feedback control method, adaptive feedback control method, time-delay method, back stepping design method, projective synchronization, sampled-data synchronization method etc. The literature review on chaos synchronization pinpoints that compared to any other method adaptive feedback control method is simple, convenient and efficient methodology to implement the chaos synchronization. In this research work, the chaos synchronization for chaotic systems is considered with unknown parameters for master and slave systems based on Lyapunon stability theorem. The synchronization of chaotic systems with unknown parameters is achieved in this paper by designing adaptive controllers and parameters updated law.

The digital circuit implementation of proposed adaptive feedback control method for chaotic system is very important for the digital information system such as digital communication system ${ }^{(30,31)}$, true random number generator $^{(32)}$, and pseudorandom number generator ${ }^{(33)}$, secure image transmission ${ }^{(34)}$, etc. Recently, many researchers focused on the Field Programmable Gate Array (FPGA) implementation of chaotic systems using many numerical methods such as Runge - Kutta, Euler algorithm, trigonometric polynomials and Adomian decomposition method etc. In ${ }^{(35)}$, novel 5D hyperchaotic system is implemented in FPGA using Runge - Kutta (RK-4) algorithm of Verilog in which chaotic system is designed with FPGA by dividing the whole system into three modules, such as RK4 solving module, data selector module, and numerical conversion module. In $^{(36)}$, S.T.Kingni et al. presented the FPGA implementation of an autonomous Josephson junction snap oscillator using a forward Euler algorithm of Verilog. In ${ }^{(37)}$, the chaotic oscillators are implemented in FPGA using numerical method based on trigonometric polynomials. In ${ }^{(38)}$, FPGA implementation of chaotic oscillator is presented by Karthikeyan et al. using Adomian decomposition method (ADM). The literature review on FPGA implementation of chaotic system using numerical methods indicating that if one does not choose the correct time-step, numerical methods may induce artificial chaos suppression or can engender the appearance of spurious solutions. In the worst case, chaotic behavior can be dead in the very short-time. Recently, many researchers presented the design of chaotic system with FPGA using automatic code generator in Matlab simulink such as DSP builder, system generator tool etc. Karthikeyan et al. ${ }^{(39)}$ proposed the FPGA implementation of chaotic system using an automatic code generator tool Xilinx system generator toolbox in Matlab simulink. With the help of this code generator any one can generate Verilog / VHDL program for chaotic system which can be downloaded directly in to FPGA. The design of chaotic system with FPGA using this method can be very simple and does not require any numerical for HDL program.

In this research work, the proposed adaptive feedback control scheme for identical new chaotic system is implemented in FPGA using Matlab simulink and Xilinx system generator tools. First, the proposed feedback control scheme is designed using Xilinx block set tools in Matlab simulink and corresponding VHDL code is downloaded from the system generator design. Then VHDL code is synthesized using Xilinx software environment. As they synthesize result, RTL schematic diagram and source consumed by adaptive feedback control scheme is obtained. The 
experimental results indicate that our proposed methodology is very simple and suitable for the digital implementation of adaptive feedback control scheme for the synchronization of identical chaotic systems.

\section{Design of 3D Chaotic System}

The new $3 \mathrm{D}$ chaotic system is described as given in the Equation. 1,

$$
\left(\begin{array}{c}
\dot{x_{1}}=a x_{1}-x_{2} x_{3}+b \\
\dot{x_{2}}=-c x_{2}+x_{1} x_{3}+d x_{3}^{2} \\
\dot{x_{3}}=-p x_{3}+x_{1} x_{2}+x_{2} x_{3}
\end{array}\right.
$$

Here $\mathrm{x}_{1}, \mathrm{x}_{2}$, and $\mathrm{x}_{3}$ are the state variables of new chaotic system (1) a, b, c, d and p are positive constant parameters. The system is chaotic when $a=2.86, b=9, c=10, d=0.5$ and $p=4$. The Lyapunov exponents for the new system (1) are calculated as $\mathrm{LE}_{1}=0.755939, \mathrm{LE}_{2}=0.060754$ and $\mathrm{LE}_{3}=-12.105507$. The corresponding Lyapunov exponents dimension is given as,

$$
D_{L}=2+\frac{L E_{1}+L E_{2}}{\left|L E_{3}\right|}=2.0675
$$

Equation 2 indicates that the attractor of new chaotic system (1) is a strange attractor with fractal dimension. The dynamics of Lyapunov exponents of the new chaotic system (1) is shown in Figure 1.

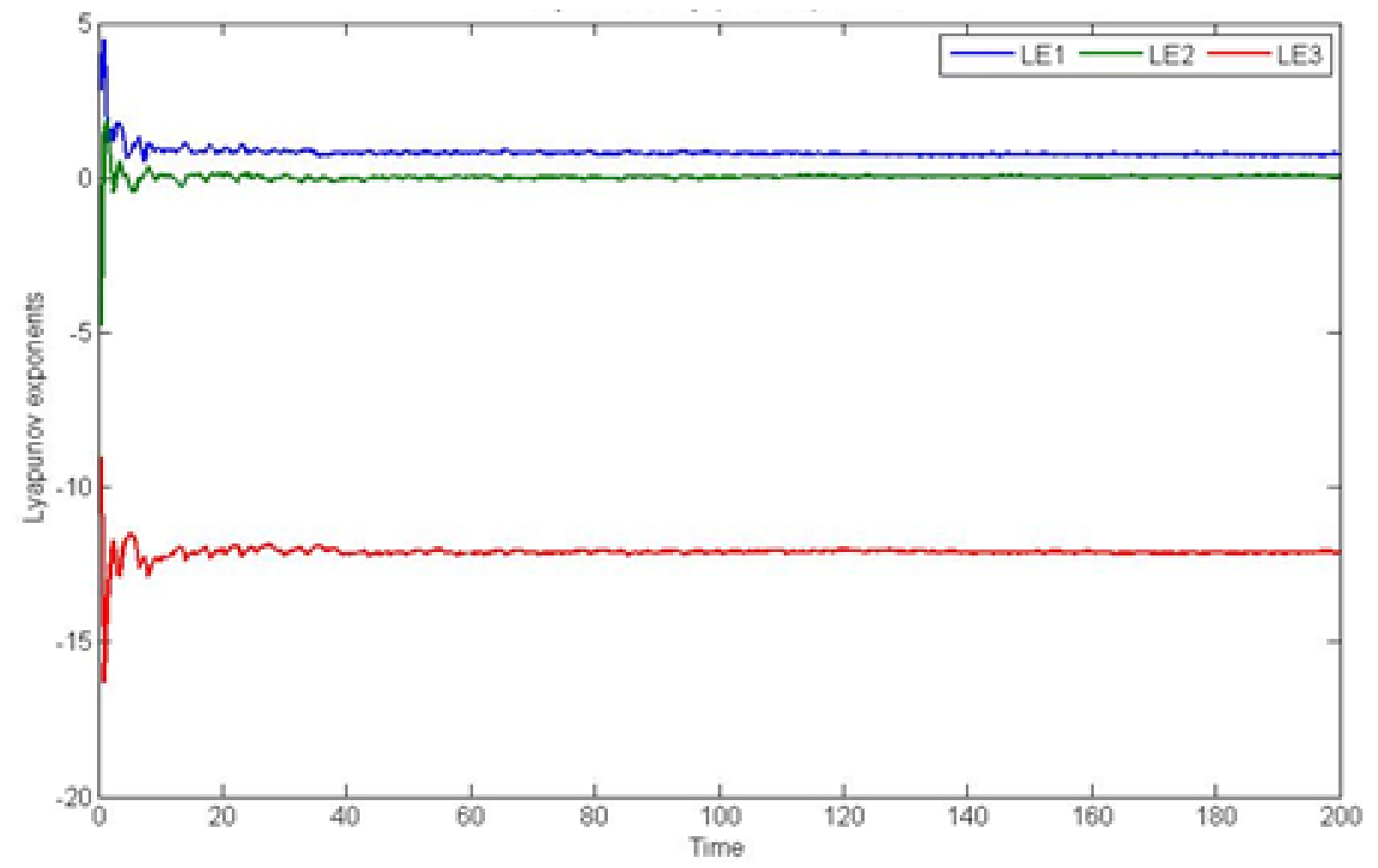

Fig 1. Dynamics of Lyapunov exponents of new system (1)

The maximal Lyapunov Exponent (MLE) of proposed chaotic system (1) is, $\mathrm{LE}_{1}=0.755939>0$ indicates that the proposed new system (1) has a chaotic behavior for the parameter values $a=2.86, b=9, c=10, d=0.5$ and $p=4$. Since the sum of the Lyapunov Exponents is negative, the new system (1) is dissipative. The new system (1) is found to be 
invariant when coordinates are transformed as, $\mathrm{S}:\left(\mathrm{x}_{1}, \mathrm{x}_{2}, \mathrm{x}_{3}\right) \rightarrow\left(-\mathrm{x}_{1}, \mathrm{x}_{2},-\mathrm{x}_{3}\right)$. This indicates that the new system (1) has symmetry about the $\mathrm{x}_{2}$ - axis. The phase portraits of new 3D chaotic system are shown in Figure $2(\mathrm{a}-\mathrm{d})$.

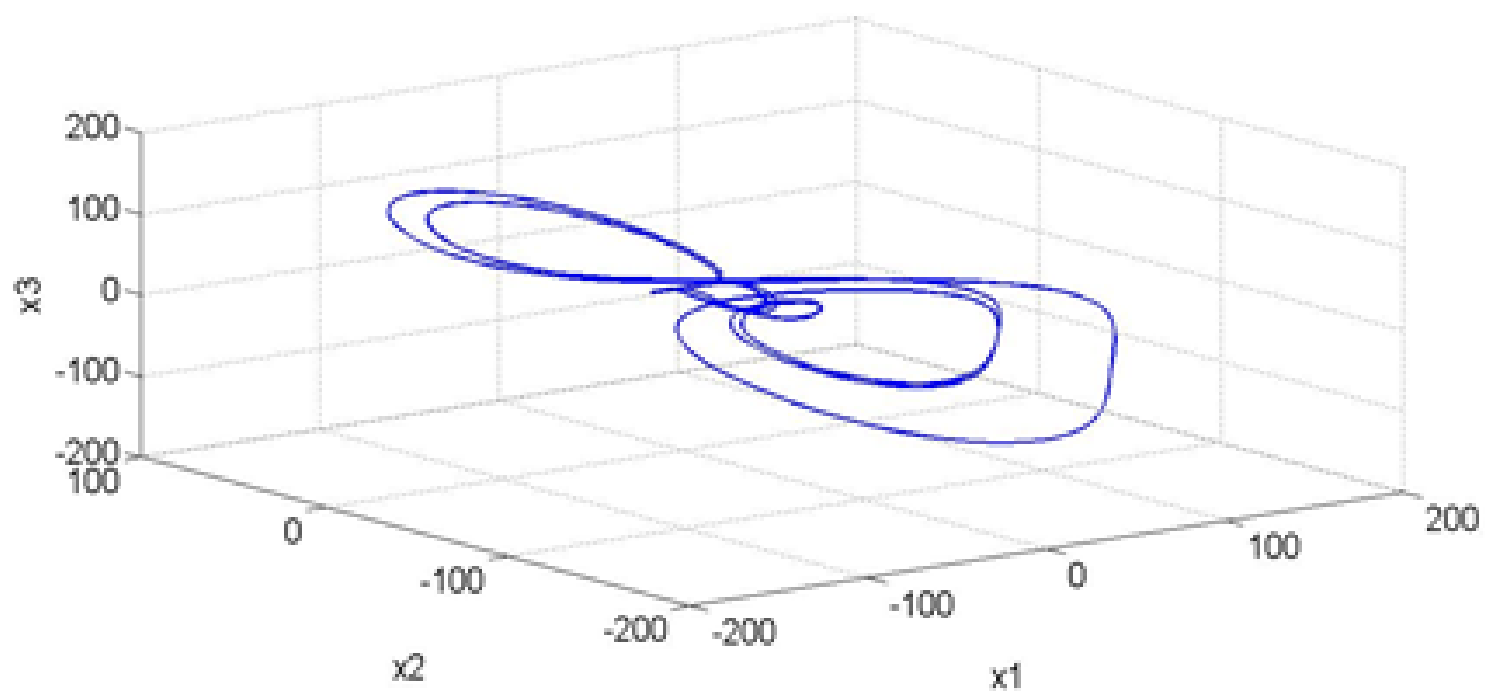

Fig 2. The phase portrait of new chaotic System (1) with initial conditions $[\mathrm{x} 1(0), \mathrm{x} 2(0), \mathrm{x} 3(0)]=[5,10,30]$

The equilibrium points of new chaotic system (1) are calculated by letting the right-hand side of Equation 1 is equal to zero. By calculation, we get the equilibrium points $S_{1}=(3.28,2.54,5.71), S_{2}=(-8.07,2.07,-8.66), S_{3}=(-$ $0.18-j 0.26,5.4-j 0.15,0.623+j 1.04)$, and $S_{4}=(0.17-j 0.01,5.4+j 0.15,0.63-j 0.01)$. The Jacobian matrix $(J)$ of the system (1) is given by Equation 3.

$$
J=\left(\begin{array}{ccc}
20 / 7 & -x_{3} & -x_{2} \\
x_{3} & -10 & x_{1}+x_{3} \\
x_{2} & x_{1}+x_{3} & x_{2}-4
\end{array}\right]
$$

The Eigen values obtained is shown in Table 1 and the system (1) is unstable at all equilibrium points $S_{1}, S_{2}, S_{3}$, and $\mathrm{S}_{4}$. The Poincare map for new chaotic system (1) for $\mathrm{a}=2.86$ is shown in Figure 3. The Poincaré map is a useful tool for analyzing the dynamical characteristics of chaotic system. In the chaotic case, the phase portrait of new chaotic system (1) is very dense in the sense that the trajectories of the motion are very close to each other. It can be only indicative of the minima and maxima of the motion. Any other characterization of the motion is difficult to be interpreted. So, one way to capture the qualitative features of the strange attractor is to obtain the Poincaré map.

Table 1. Eigen values of Jacobian matrix

\begin{tabular}{lllll}
\hline Equilibrium Points & $\lambda_{1}$ & $\lambda_{2}$ & $\lambda_{3}$ & Comments \\
\hline & & & & \\
$S_{1}$ & $3.23+\mathrm{j} 5.2$ & $3.23-\mathrm{j} 5.2$ & -15.1 & Unstable \\
$S_{2}$ & -24.83 & 1.86 & 13.9 & Unstable \\
$S_{3}$ & $2.2+\mathrm{j} 5.2$ & $2.04-\mathrm{j} 5.4$ & -10 & Unstable \\
$S_{4}$ & $2.3-\mathrm{j} 5.3$ & $2+\mathrm{j} 5.5$ & -10 & Unstable \\
\hline
\end{tabular}




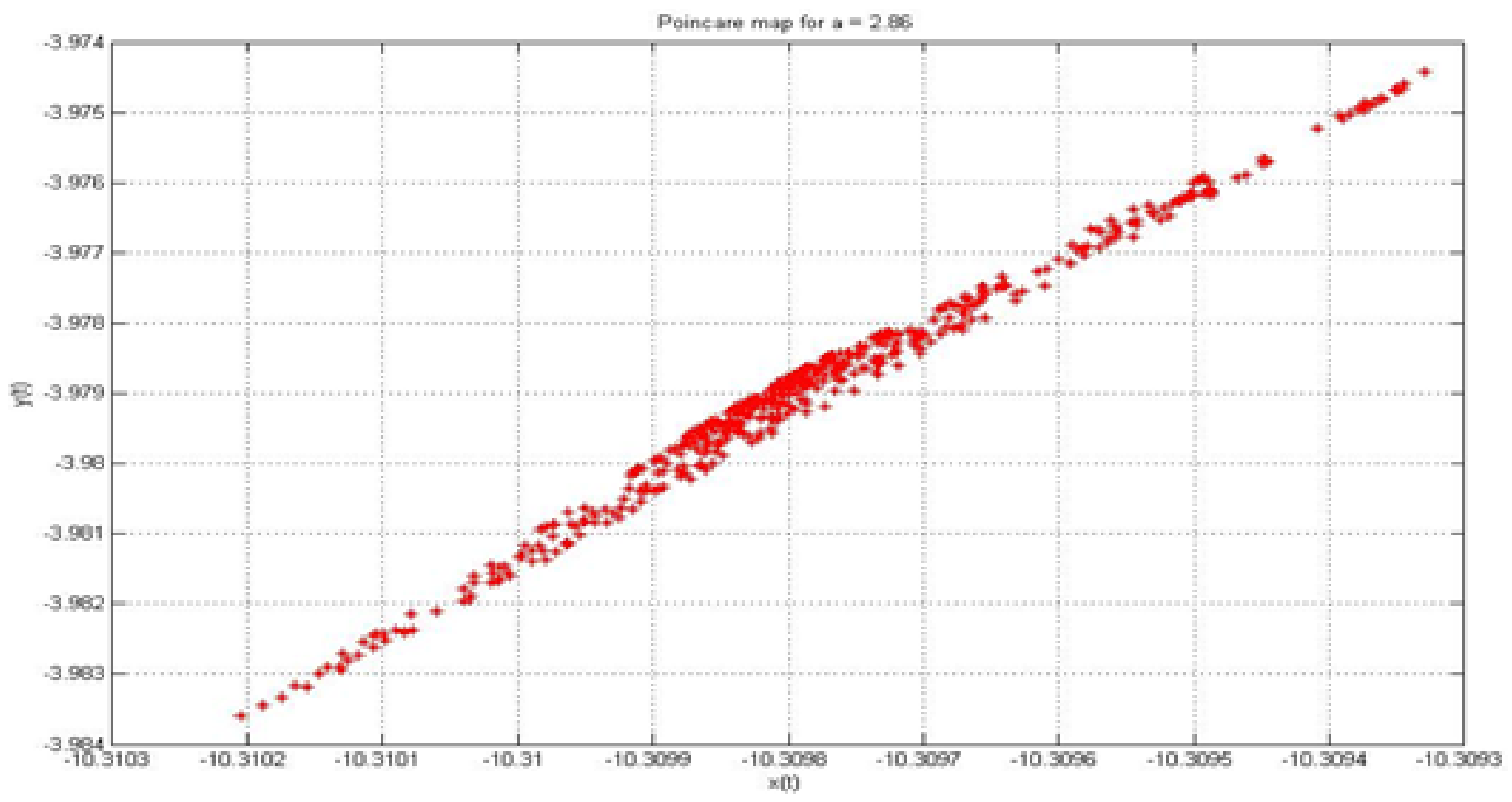

Fig 3. Poincare map for $\mathrm{a}=2.86$

\section{Adaptive Feedback Control for New 3 - D Chaotic System}

In this section, based on the adaptive feedback control theory, the complete synchronization between two identical new chaotic systems is achieved using master - slave formalization. According to that Equation 1 is considered as a master chaotic system and the slave chaotic system is given by the Equation 4 .

$$
\left(\begin{array}{c}
\dot{y_{1}}=a y_{1}-y_{2} y_{3}+b+u_{1} \\
\dot{y_{2}}=-c y_{2}+y_{1} y_{3}+d y_{3}^{2}+u_{2} \\
\dot{y_{3}}=-p y_{3}+y_{1} y_{2}+y_{2} y_{3}+u_{3}
\end{array}\right.
$$

Here $y_{1}, y_{2}, y_{3}$ are the state variables of slave system, a, b, c, d, and $\mathrm{p}$ are positive constant parameters and $u_{1}, u_{2}$, and $u_{3}$ are control functions. Our goal is to determine the control function from adaptive feedback control method. The complete synchronization error are defined as,

$$
\left(\begin{array}{l}
e_{1}=y_{1}-x_{1} \\
e_{2}=y_{2}-x_{2} \\
e_{3}=y_{3}-x_{3}
\end{array}\right.
$$

The synchronization of error dynamics is obtained as,

$$
\left(\begin{array}{c}
\dot{e}_{1}=a e_{1}-y_{2} y_{3}+x_{2} x_{3}+u_{1} \\
\dot{e}_{2}=-c e_{2}+y_{1} y_{3}-x_{1} x_{3}+d\left(y_{3}^{2}-x_{3}^{2}\right)+u_{2} \\
\dot{e}_{3}=-p e_{3}+y_{1} y_{2}+y_{2} y_{3}-x_{1} x_{2}-x_{2} x_{3}+u_{3}
\end{array}\right.
$$


The Controller $u$ is derived for the adaptive feedback control functions such that the error drives to zero. The adaptive feedback controller is given in Equation 7.

$$
\left(\begin{array}{c}
u_{1}=-\widehat{a} e_{1}+y_{2} y_{3}-x_{2} x_{3}-k_{1} e_{1} \\
u_{2}=\widehat{c} e_{2}-y_{1} y_{3}+x_{1} x_{3}-\widehat{d}\left(y_{3}^{2}-x_{3}^{2}\right)-k_{2} e_{2} \\
u_{3}=\widehat{p} e_{3}-y_{1} y_{2}-y_{2} y_{3}+x_{1} x_{2}+x_{2} x_{3}-k_{3} e_{3}
\end{array}\right.
$$

Here $\widehat{a}, \widehat{c}, \widehat{d}$ and $\widehat{p}$ are the estimate values of the unknown parameters $\mathrm{a}, \mathrm{b}, \mathrm{c}, \mathrm{d}$ and $\mathrm{p}$ respectively. Then by substituting Equation 7 in Equation 6, the error dynamics is obtained as in Equation 8.

$$
\left(\begin{array}{c}
\dot{e}_{1}=e_{a} e_{1}-k_{1} e_{1} \\
\dot{e}_{2}=-e_{c} e_{2}+e_{d}\left(y_{3}^{2}-x_{3}^{2}\right)-k_{2} e_{2} \\
\dot{e}_{3}=-e_{p} e_{3}-k_{3} e_{3}
\end{array}\right.
$$

Here the estimation error for unknown parameter are given as,

$$
\left(\begin{array}{l}
e_{a}=a-\widehat{a} \\
e_{c}=c-\widehat{c} \\
e_{d}=d-\widehat{d} \\
e_{p}=p-\widehat{p}
\end{array}\right.
$$

By differentiating Equation 9,

$$
\left(\begin{array}{rl}
\dot{e}_{a} & =-\dot{\hat{a}} \\
\dot{e}_{c} & =-\dot{\hat{c}} \\
\dot{e}_{d} & =-\dot{\widehat{d}} \\
\dot{e}_{p} & =-\dot{\hat{p}}
\end{array}\right.
$$

Consider a Lyapunov function candidate as,

$$
\dot{V}=e_{1} \dot{e}_{1}+e_{2} \dot{e}_{2}+e_{3} \dot{e}_{3}+e_{a} \dot{e}_{a}+e_{c} \dot{e}_{c}+e_{d} \dot{e}_{d}+e_{p} \dot{e}_{p}
$$

By substituting Equation 8 and Equation 10 in Equation 11,

$$
\begin{aligned}
& \dot{V}=e_{a}\left(e_{1}^{2}-\dot{\hat{a}}\right]+e_{c}\left(-e_{2}^{2}-\dot{\hat{c}}\right]+e_{d}\left(e_{2}\left(y_{3}^{2}-x_{3}^{2}\right)-\dot{\hat{d}}\right]+e_{p}\left(-e_{3}^{2}-\dot{\hat{p}}\right]-\left(k_{1} e_{1}^{2}+k_{2} e_{2}^{2}+k_{3} e_{3}^{2}\right) \\
& \dot{V}=-\left(k_{1} e_{1}^{2}+k_{2} e_{2}^{2}+k_{3} e_{3}^{2}\right)
\end{aligned}
$$

Let us choose the parameter update law as,

$$
\left(\begin{array}{c}
\dot{e}_{a}=-\dot{\widehat{a}}=e_{1}^{2} \\
\dot{e}_{c}=-\dot{\widehat{c}}=-e_{2}^{2} \\
\dot{e}_{d}=-\dot{\widehat{d}}=e_{2}\left(y_{3}^{2}-x_{3}^{2}\right) \\
\dot{e}_{p}=-\dot{\hat{p}}=-e_{3}^{2}
\end{array}\right.
$$

The Equation 12 indicates that by Lyapunov stability theory, it is determined that the synchronization error $e_{i}, i=$ $1,2,3$ and the parameter estimation error $e_{a}, e_{c}, e_{d}, e_{p}$ decay to zero exponentially with time. Thus the adaptive controlled slave chaotic system is given as.

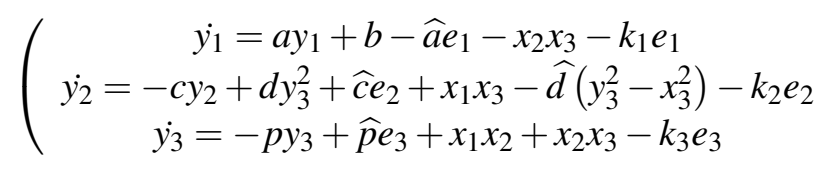




\section{Digital Circuit Realization on FPGA of Adaptive Feedback Control for New 3 - D Chaotic System}

In this section, the digital circuit realization of proposed adaptive feedback control methodology is achieved in FPGA Virtex4 xc4vfx100-12ff1152. The new 3 - D chaotic system corresponding to Equation 1, adaptive controllers corresponding to Equation 7 and parameter update laws corresponding to Equation 13 are constructed using MATLAB Simulink and Xilinx system generator block sets. Figure 4 represents the FPGA implementation of new 3 D chaotic system using Xilinx block set. Figure $5(\mathrm{a}-\mathrm{c})$ represents the FPGA implementation of adaptive controllers $\mathrm{u}_{1}, \mathrm{u}_{2}$, and $\mathrm{u}_{3}$ using Xilinx block set respectively. Figure 6 represents the FPGA implementation of parameter update law in Xilinx block set. Figure 7 represents the FPGA implementation of proposed adaptive feedback control for new 3D chaotic system using Xilinx System Generator blocks. The master subsystem contains the new master chaotic generator ( Figure 4), the slave subsystem contains the block diagram of slave system given in Equation 4 . The parameter update law subsystem contains the Figure 6 and the control commands subsystem contains the Figure 5. This FPGA implementation is adopted with a fixed point and with a representation of the real data on 32 bits (12Q20), 12 for the entire and 20 for the fraction.

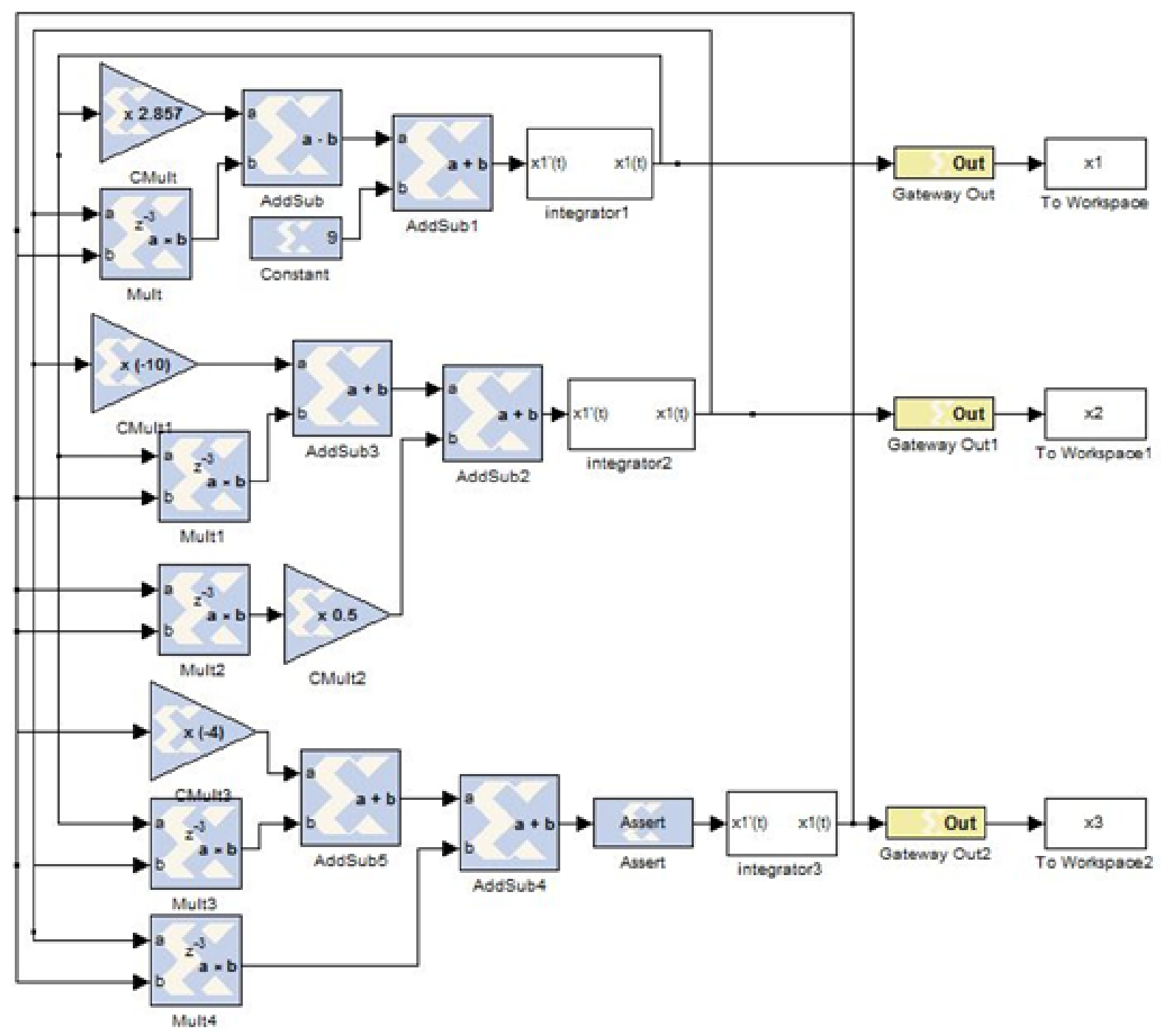

Fig 4. FPGA Implementation of new chaotic system (1) 


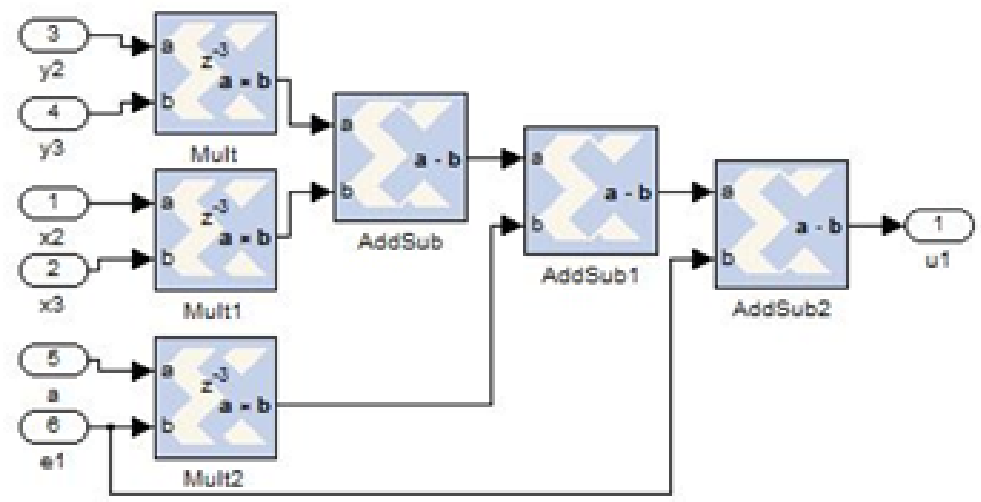

(a) Implementation of $\mathrm{U}_{1}$

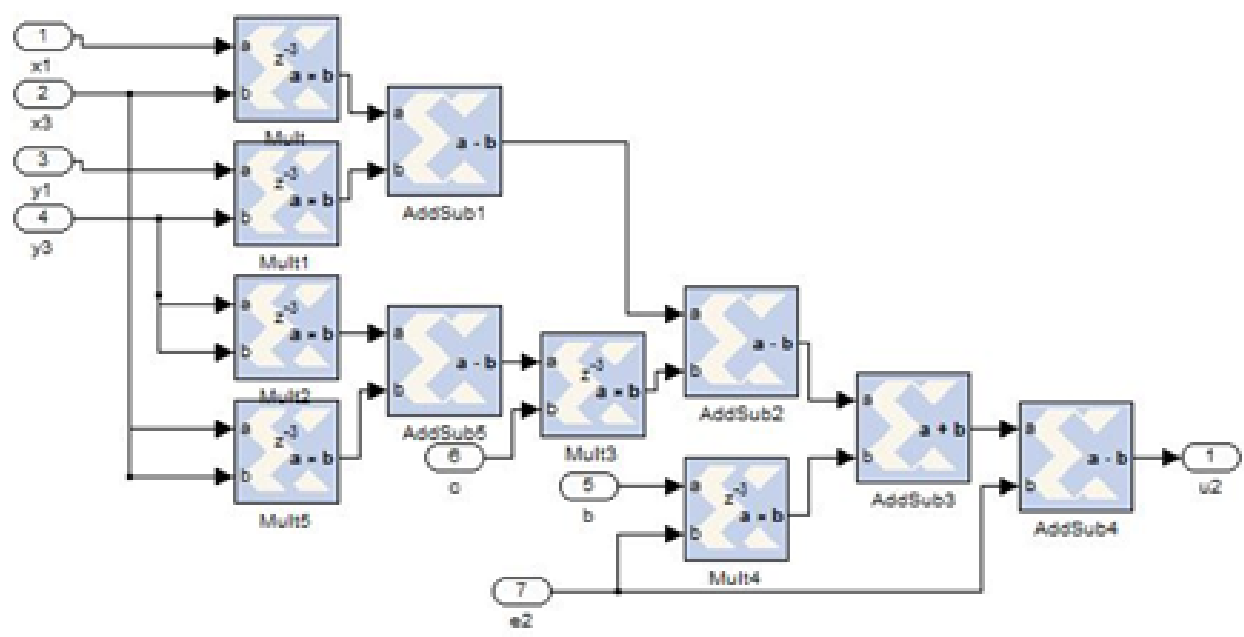

(b) Implementation of $\mathrm{U}_{2}$

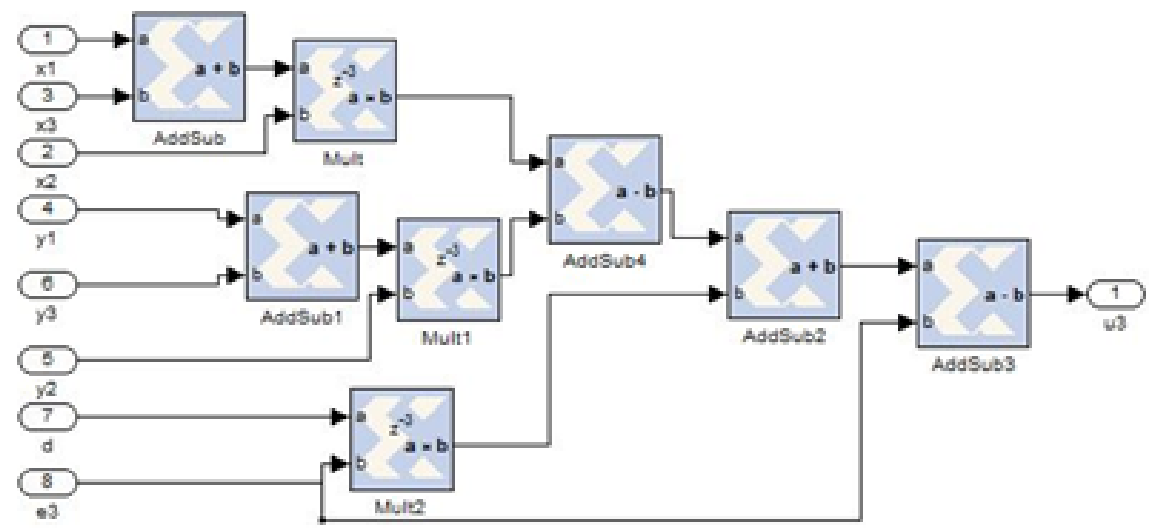

(c) Implementation of $\mathrm{U}_{3}$

Fig 5. FPGA Implementation of adaptive controllers 


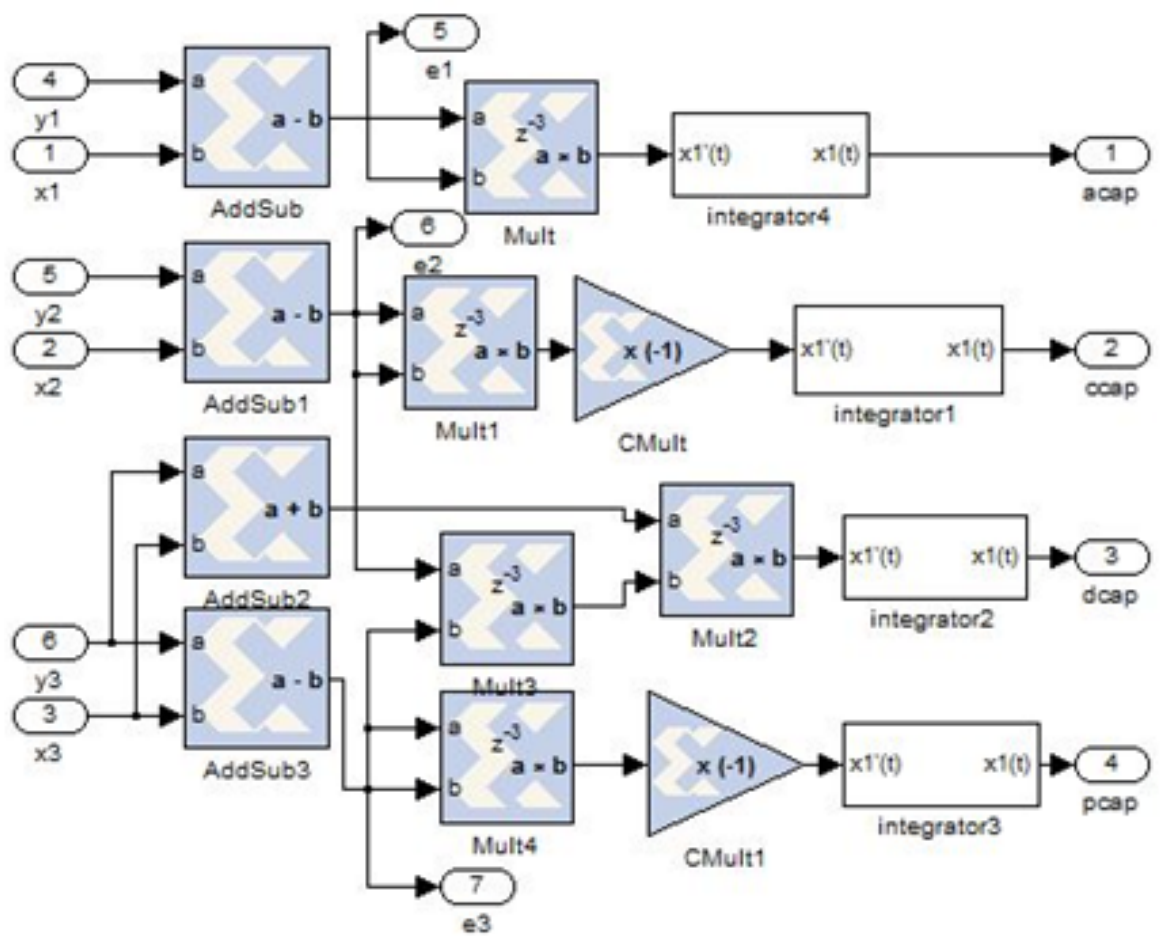

Fig 6. FPGA Implementation of unknown parameters

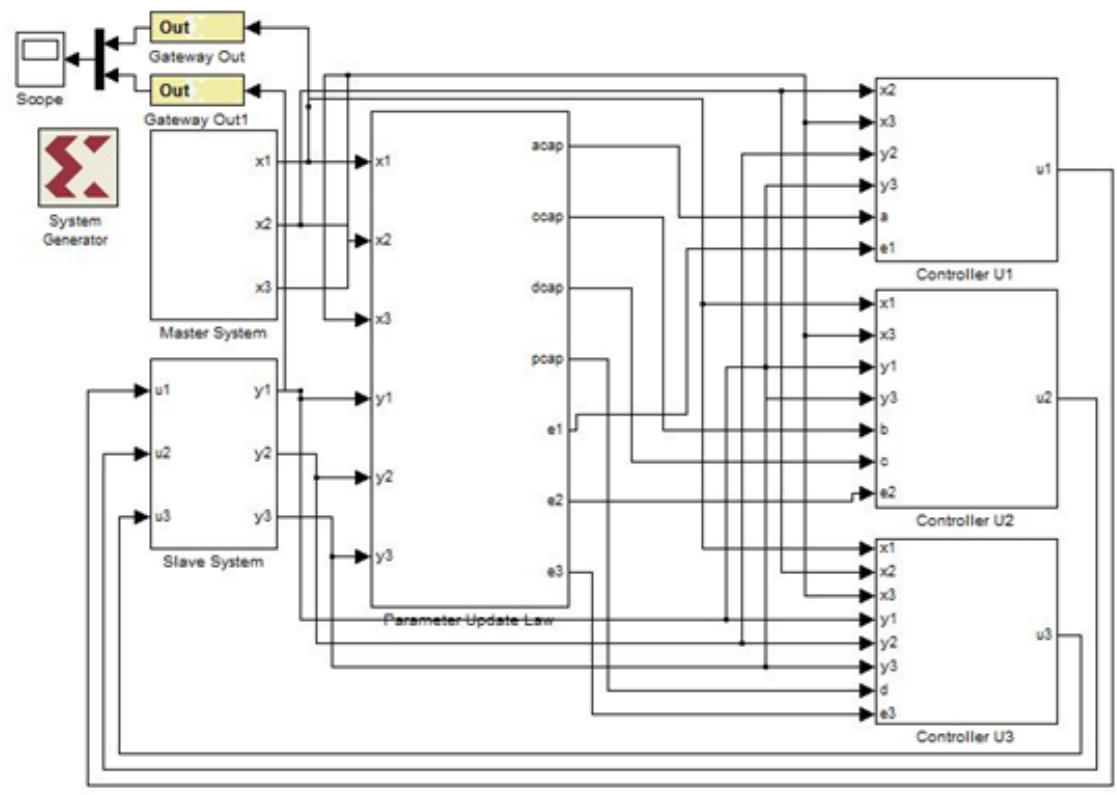

Fig 7. FPGA Implementation of adaptive feedback control scheme for new chaoticsystem

The initial conditions for master chaotic system are chosen as $x_{1}(0)=5, x_{2}(0)=10$ and $x_{3}(0)=30$ and the initial conditions are feed up in the integrator block in Figure 4. The initial conditions for the state variables of slave system can be chosen as $y_{1}(0)=20, y_{2}(0)=12$ and $y_{3}(0)=30$. We adopt this FPGA implementation of adaptive 
feedback control method with a fixed point and with a representation of the real data on 32 bits (12Q20), 12 for the entire and 20 for the fraction. Figure 8 represents the time evolutions of the master and controlled slave system variables. Figure 9 represents the time history of error signals which reaches zero when slave system is controlled by master chaotic system. Figure 10 represents the time history of adaptive controller signals which also reaches zero when slave system is controlled by master chaotic system.

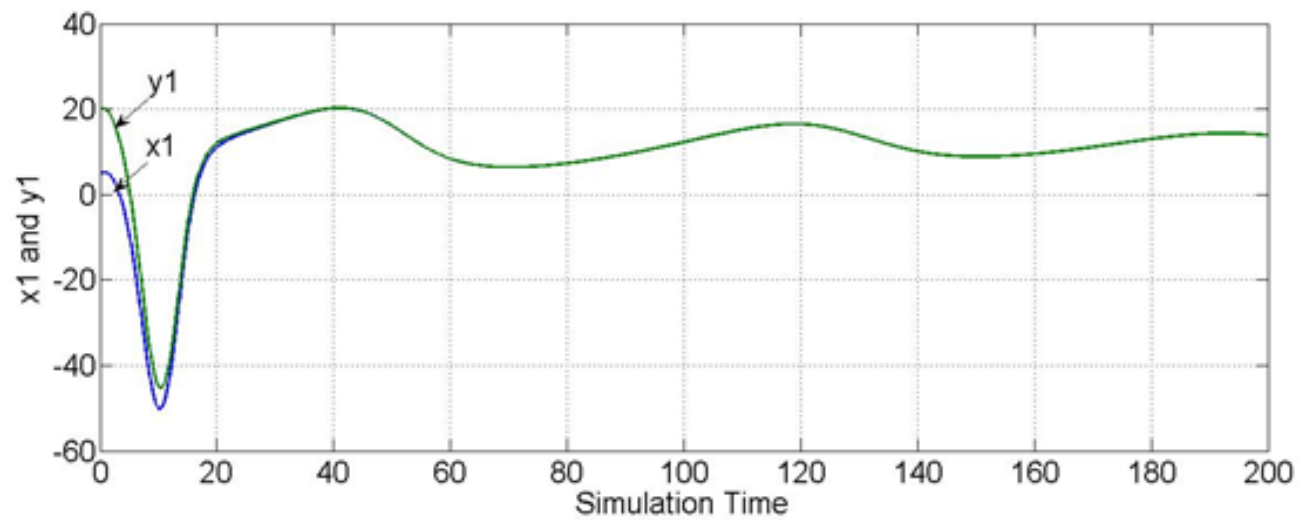

(a)

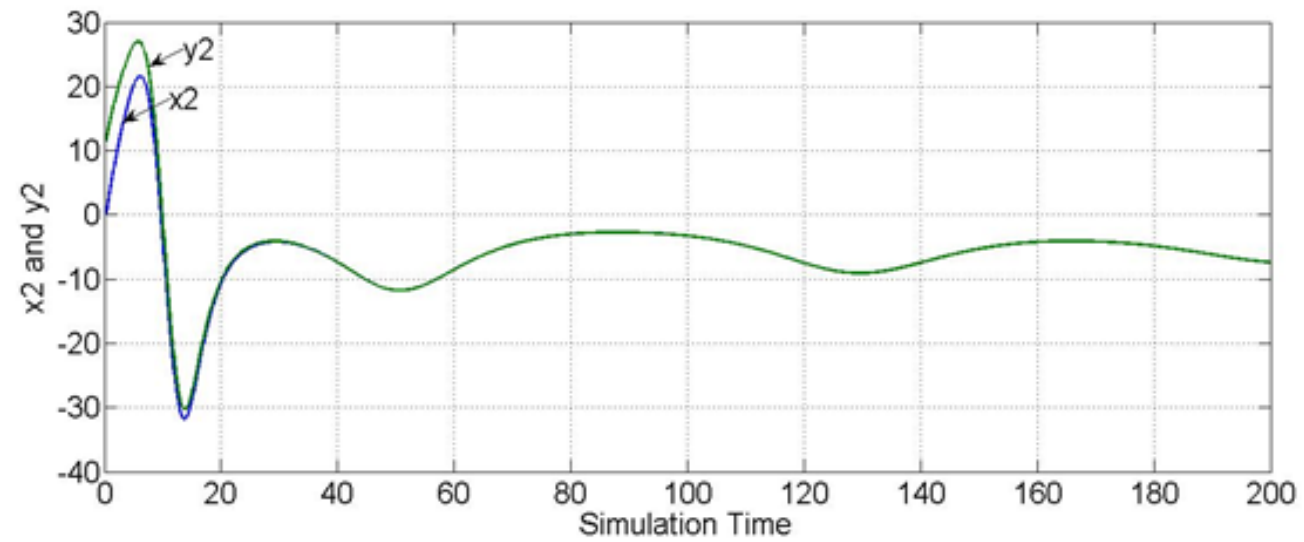

(b)

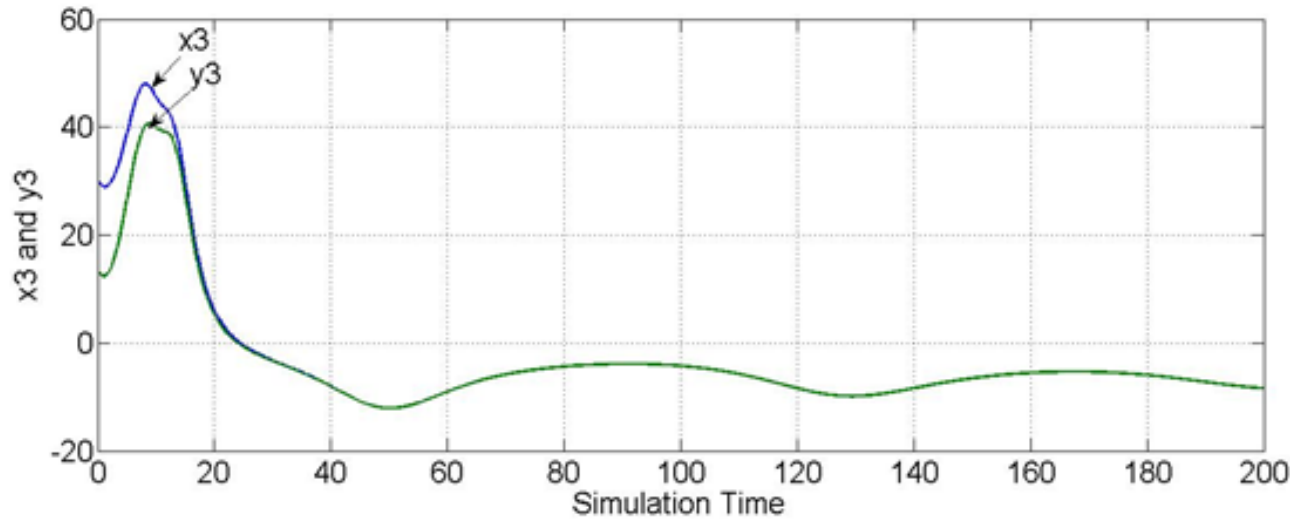

(c)

Fig 8. Time History of the master and controlled slave system variables (a) $x_{1}-y_{1}$, (b) $x_{2}-y_{2}$, and (c) $x_{3}-y_{3}$ 


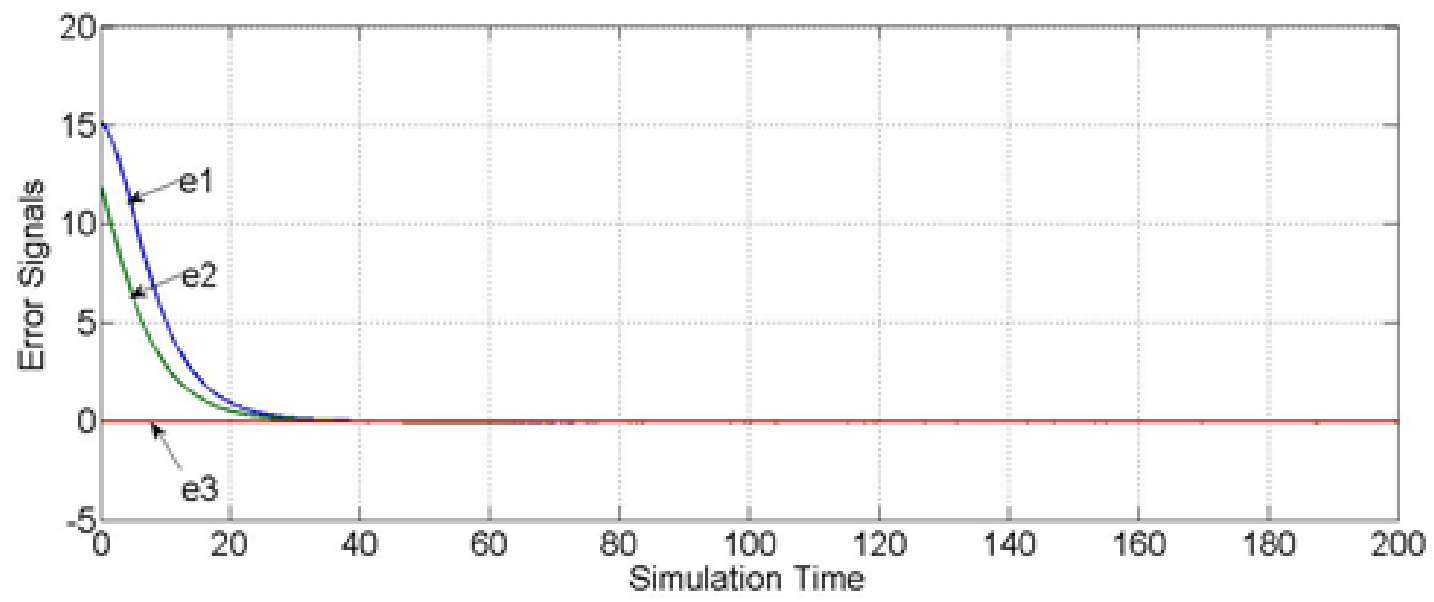

Fig 9. Time history of error signals

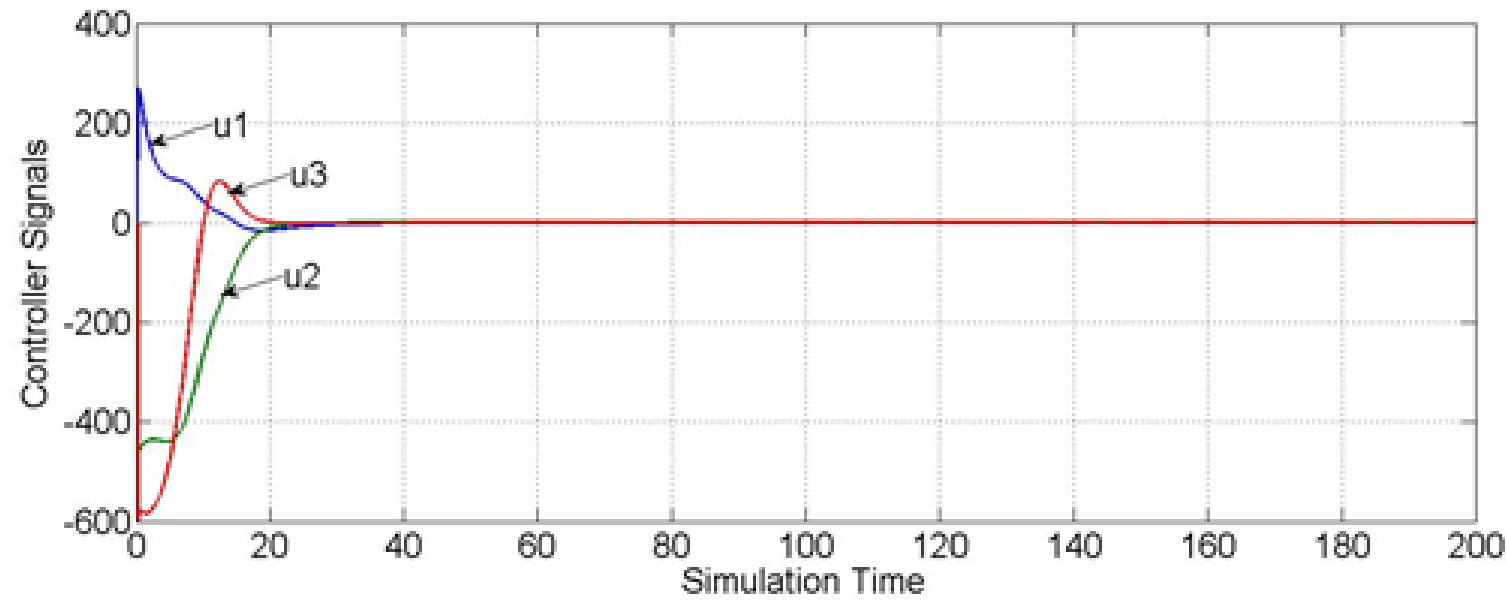

Fig 10. Time history ofadaptive controllers

After that, VHDL code for completely controlled $3 \mathrm{D}$ chaotic systems is downloaded from the MATLAB Simulink design shown in Figure 7. Then the VHDL code is simulated using Xilinx software and the source consumed by the controlled $3 \mathrm{D}$ chaotic system is obtained as given in Table 2. Also, RTL schematic diagram for controlled $3 \mathrm{D}$ chaotic system is generated as shown in Figure 11.

Table 2. Source consumed controlled new chaotic system

\begin{tabular}{llll}
\hline Slice Logic Utilization & Source consumed by controlled new chaotic systems & Percentage \\
& Used & Available & 1 \\
No. of slice registers & 1064 & 393600 & 1 \\
No. of slice LUTs & 3091 & 196800 & 1 \\
No. of occupied slices & 949 & 49200 & 48 \\
No. of bonded IOBs & 289 & 600 & 4 \\
\hline
\end{tabular}




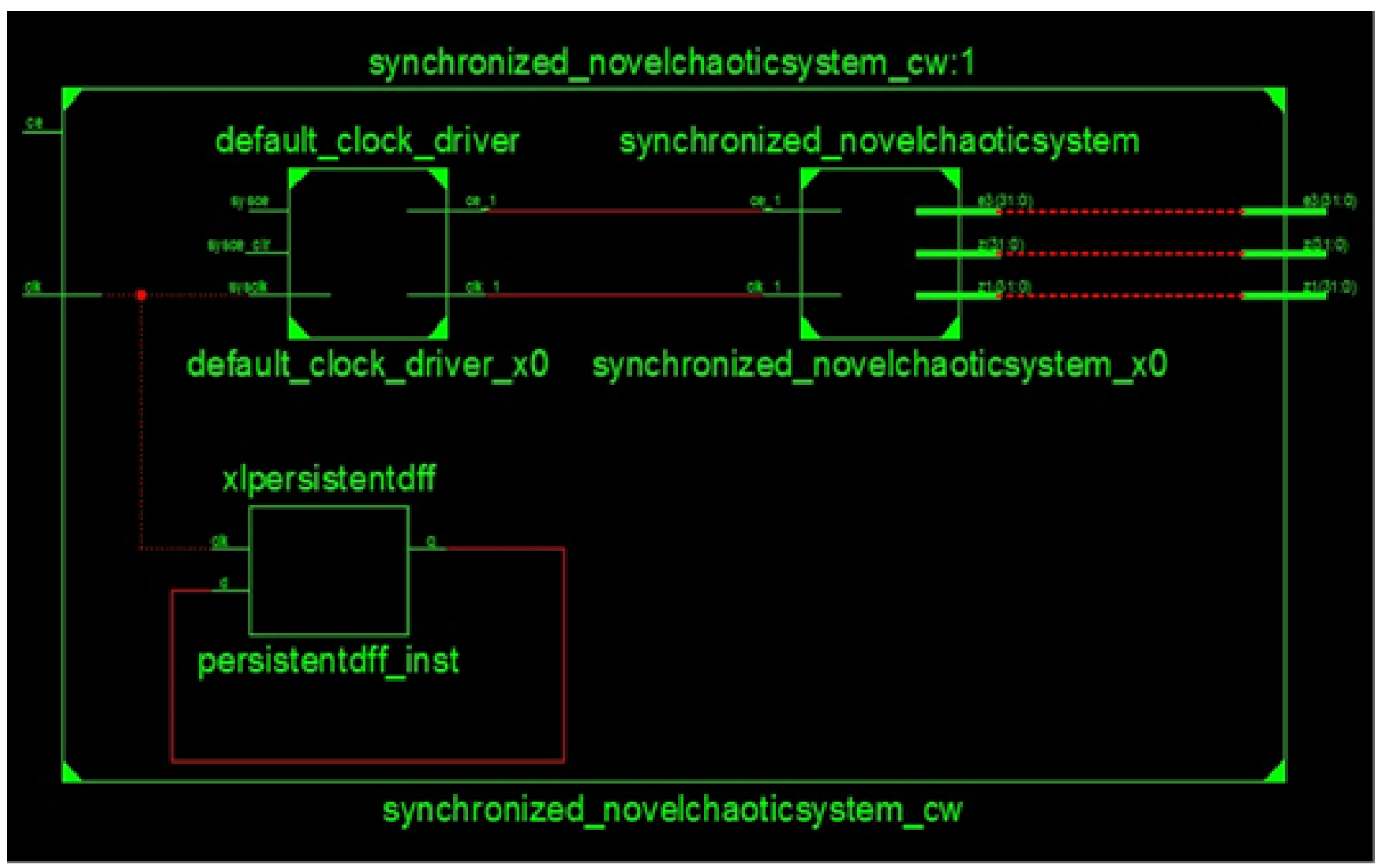

Fig 11. RTL schematic diagramof controlled chaotic system

\section{Conclusion}

In this paper, we proposed a new chaotic system and its properties are analyzed in detail. The synchronization of new identical chaotic system is derived using master - slave adaptive feedback control methodology. The experimental results and numerical simulations proved that the proposed feedback methodology is efficient and convenient for the chaos synchronization. Further, the digital implementation of proposed adaptive feedback control scheme is achieved on FPGA using automatic VHDL code generator such as Xilinx system generator. The Matlab simulation results and Xilinx simulation results are indicating that the proposed methodology is very simple, effective and convenient for the digital implementation of adaptive feedback control method for chaotic systems.

\section{References}

1) Jafari S, Rajagopal K, Hayat T, Alsaedi A, Pham VT. Simplest Megastable Chaotic Oscillator. International Journal of Bifurcation and Chaos. 2019;29(13):1950187-1950187. Available from: https://dx.doi.org/10.1142/s0218127419501876. doi:10.1142/s0218127419501876.

2) Wang X, Yu J, Jin C, Ching H, Yu S. Chaotic oscillator based on memcapacitor and meminductor. Nonlinear Dynamics. 2019;96:161-173. Available from: https://doi.org/10.1007/s11071-019-04781-5.

3) Sun J, Yang Q, Wang Y. Dynamical analysis of novel memristor chaotic system and DNA encryption application. Iranian Journal of Science and Technology, Transactions of Electrical Engineers. 2020;44:449-460. Available from: https://doi.org/10.1007/s40998-019-00239-x.

4) Arab A, Rostami MJ, Ghavami B. An image encryption method based on chaos system and AES algorithm. The Journal of Supercomputing. 2019;75(10):6663-6682. Available from: https://dx.doi.org/10.1007/s11227-019-02878-7. doi:10.1007/s11227-019-02878-7.

5) Singh PP, Roy BK. Microscopic chaos control of chemical reactor system using non linear active plus proportional integral sliding mode control techniques. The European Physical Journal Special Topics. 2019;228(1):169-184.

6) Vaidhyanathan S, Boulkroune A. 2016. Available from: https://doi.org/10.1007/978-3-319-30279-9_19. 
7) Cui S, Zhang J. Chaotic secure communication based on single feedback phase modulation and channel transmission. IEEE Photonics Journal. 2019;11(5):1-8.

8) Wang DM, Wang LS, Guo YY, Wang YC, Wang AB. Key space enhancement of optical chaos secure communication: chirped FBG feedback semiconductor laser. Optics Express. 2019;27(3):3065-3065. Available from: https://dx.doi.org/10.1364/oe.27.003065. doi:10.1364/oe.27.003065.

9) Vaidyanathan S. Adaptive control of the FitzHugh-Nagumo chaotic neuron model. International Journal of PharmTech Research. 2015;8(6):117-127.

10) Vaidyanathan S. Adaptive chaotic synchronization of enzymes-substrates system with ferroelectric behaviour in brain waves. International Journal of PharmTech Research. 2015;8(5):964-973.

11) Pecora LM, Carroll TL. Synchronization in chaotic systems. Physical Review Letters. 1990;64(8):821-824. Available from: https://dx.doi. org/10.1103/physrevlett.64.821. doi:10.1103/physrevlett.64.821.

12) Fang JS, Tsai JSH, Yan JJ, Guo SM. Adaptive Chattering-Free Sliding Mode Control of Chaotic Systems with Unknown Input Nonlinearity via Smooth Hyperbolic Tangent Function. Mathematical Problems in Engineering. 2019;2019:1-9. Available from: https://dx.doi.org/10. 1155/2019/4509674. doi:10.1155/2019/4509674.

13) Pérez-Cruz JH, Tamayo-Meza PA, Figueroa M, Silva-Ortigoza R, Ponce-Silva M, Rivera-Blas R, et al. Exponential Synchronization of Chaotic Xian System Using Linear Feedback Control. Complexity. 2019;2019:1-10. Available from: https://dx.doi.org/10.1155/2019/ 4706491. doi:10.1155/2019/4706491.

14) Hannachi F. Analysis, dynamics and adaptive control synchronization of a novel chaotic 3-D system. SN Applied Sciences. 2019;1(2). Available from: https://dx.doi.org/10.1007/s42452-019-0175-3. doi:10.1007/s42452-019-0175-3.

15) Khan A, Budhraja M, Ibraheem A. Synchronization Among Different Switches of Four Non-identical Chaotic Systems via Adaptive Control. Arabian Journal for Science and Engineering. 2019;44(3):2717-2728. Available from: https://dx.doi.org/10.1007/s13369-0183458-x. doi:10.1007/s13369-018-3458-x.

16) Vaidyanathan S, Abba O, Gambo B, Alidou M. A new three dimensional chaotic system: its adaptive control and circuit design. International Journal of Automation and Control. 2019;13(1):101-121.

17) Hamed T. 2017. Available from: https://doi.org/10.1515/nleng-2017-0050.

18) Singh AK, Yadav VK, Das S. Synchronization of Time-delay Chaotic Systems with Uncertainties and External Disturbances. The interdisciplinary journal of Discontinuity, Nonlinearity, and Complexity. 2019;8(1):13-21. Available from: https://dx.doi.org/10.5890/dnc. 2019.03.002. doi:10.5890/dnc.2019.03.002.

19) Vaidyanathan S, Sampath S. Anti-synchronization of four-wing chaotic systems via sliding mode control. International Journal of Automation and Computing. 2012;9(3):274-279. Available from: https://dx.doi.org/10.1007/s11633-012-0644-2. doi:10.1007/s11633012-0644-2.

20) Vaidyanathan S. Synchronization of Tokamak systems with symmetric and magnetically confined plasma via adaptive control. International Journal of ChemTech Research. 2015;8(6):818-827.

21) Vaidyanathan S. Adaptive biological control of generalized Lotka-Volterra three-species biological system. International Journal of PharmTech Research. 2015;8(4):622-631.

22) Cun FF, Yan RT, Ying HW, Hai YY. Active backstepping control of projective synchronization among different non linear systems. Journal of Control, Measurements, Electronics, computing and communications. 2019;58:295-301.

23) Eroglu D, Lamb JSW, Pereira T. Synchronisation of chaos and its applications. Informa UK Limited. 2017. Available from: https: //dx.doi.org/10.1080/00107514.2017.1345844. doi:10.1080/00107514.2017.1345844.

24) Chen L, Huang C, Liu H, Xia Y. Anti synchronization of a class of chaotic systems with applications to Lorenz system: A unified analysis of integer order and Fractional order. Mathematics. 2019;7.

25) Chai $X$, Huigan Z. Function projective lag synchronization of chaotic systems with certain parameters via adaptive - impulsive control. International Journal of Automation and computing. 2019;16:238-247.

26) Vaidyanathan S, Sambas A, Kacar S, Cavusoglu U. A New Finance Chaotic System, its Electronic Circuit Realization, Passivity based Synchronization and an Application to Voice Encryption. Nonlinear Engineering. 2019;8(1):193-205. Available from: https://dx.doi.org/ 10.1515/nleng-2018-0012. doi:10.1515/nleng-2018-0012.

27) Lee SH, Kapila V, Porfiri M, Panda A. Master-slave synchronization of continuously and intermittently coupled sampled-data chaotic oscillators. Communications in Nonlinear Science and Numerical Simulation. 2010;15(12):4100-4113. Available from: https://dx.doi.org/ 10.1016/j.cnsns.2010.01.035. doi:10.1016/j.cnsns.2010.01.035.

28) Pakiriswamy S. Active Controller Design for the Generalized Projective Synchronization Of Three-Scroll Chaotic Systems. International Journal of Advanced Information Technology. 2012;2(1):37-53. Available from: https://dx.doi.org/10.5121/ijait.2012.2104. doi:10.5121/ijait.2012.2104.

29) Sundarapandian V. Sliding mode controller design for global chaos synchronization of Rucklidge Chaotic Systems. International Journal on Bioinformatics and Biosciences. 2012;2(4):23-31.

30) Kaddoum G. Wireless Chaos-Based Communication Systems: A Comprehensive Survey. IEEE Access. 2016;4:2621-2648. Available from: https://dx.doi.org/10.1109/access.2016.2572730. doi:10.1109/access.2016.2572730.

31) N, Quyen X, Kyamakya K. Springer. 2018. Available from: https://doi.org/10.1007/978-3-319-58996-1_11.

32) Bonilla LL, Alvaro M, Carretero M. Chaos-based true random number generators. Journal of Mathematics in Industry. 2016;7(1). 
Available from: https://dx.doi.org/10.1186/s13362-016-0026-4. doi:10.1186/s13362-016-0026-4.

33) Lynnyk V, Sakamoto N, Čelikovský S. Pseudo random number generator based on the generalized Lorenz chaotic system. Elsevier BV. 2015. Available from: https://dx.doi.org/10.1016/j.ifacol.2015.11.046. doi:10.1016/j.ifacol.2015.11.046.

34) Guillén-Fernández O, Meléndez-Cano A, Tlelo-Cuautle E, Núñez-Pérez JC, de Jesus Rangel-Magdaleno J. On the synchronization techniques of chaotic oscillators and their FPGA-based implementation for secure image transmission. PLOS ONE. 2019;14(2):e0209618e0209618. Available from: https://dx.doi.org/10.1371/journal.pone.0209618. doi:10.1371/journal.pone.0209618.

35) Yu F, Liu L, He B, Huang Y, Shi C, Cai S, et al. Analysis and FPGA Realization of a Novel 5D Hyperchaotic Four-Wing Memristive System, Active Control Synchronization, and Secure Communication Application. Complexity. 2019;2019:1-18. Available from: https: //dx.doi.org/10.1155/2019/4047957. doi:10.1155/2019/4047957.

36) Kingni ST, Rajagopal K, Tamba VK, Ainamon C, Orou JBC. Analysis and FPGA implementation of an autonomous Josephson junction snap oscillator. The European Physical Journal B. 2019;92(10). Available from: https://dx.doi.org/10.1140/epjb/e2019-100304-x. doi:10.1140/epjb/e2019-100304-x.

37) Pano-Azucena AD, Tlelo-Cuautle E, Rodriguez-Gomez G, de la Fraga LG. FPGA-based implementation of chaotic oscillators by applying the numerical method based on trigonometric polynomials. AIP Advances. 2018;8(7):075217-075217. Available from: https://dx.doi. org/10.1063/1.5038583. doi:10.1063/1.5038583.

38) Rajagopal K, Kingni ST, Khalaf AJM, Shekofteh Y, Nazarimehr F. Coexistence of attractors in a simple chaotic oscillator with fractionalorder-memristor component: analysis, FPGA implementation, chaos control and synchronization. Springer Science and Business Media LLC. 2019. Available from: https://dx.doi.org/10.1140/epjst/e2019-900001-8. doi:10.1140/epjst/e2019-900001-8.

39) Rajagopal K, Guessas L, Vaidyanathan S, Karthikeyan A, Srinivasan A. 2017. Available from: https://doi.org/10.1155/2017/7307452. 